\title{
A Conceptual Framework for Modeling and Design of Cyber-Physical Systems
}

\author{
Ioan DUMITRACHE ${ }^{1}$, Ioan Stefan SACALA ${ }^{1 *}$, Mihnea Alexandru MOISESCU ${ }^{1}$, \\ Simona Iuliana CARAMIHAI ${ }^{1}$ \\ ${ }^{1}$ University "Politehnica" of Bucharest \\ 313 Splaiul Independentei, Bucharest, Romania \\ ioan.sacala@acse.pub.ro,mihnea.moisescu@upb.ro \\ (*Corresponding author)
}

\begin{abstract}
Systems that can tightly integrate physical with virtual components have represented a priority of research and development in the area of ICT. An intensive work has been concentrated in different domains, such as: Internet of Things, Internet of Services and lately in the domain of Cyber Physical Systems whose important driver is represented by the largescale integration of the physical and cyber worlds. Authors propose a Generic System Architecture, taking into account the paradigm of Cyber-Physical Systems with a main emphasis on the future agricultural enterprise (intelligent/smart farm) as a complex system, addressing sustainability and adaptability towards environmental and market changes.
\end{abstract}

Keywords: Cyber Physical Systems, Internet of Things, Intelligent Integrated System, Intelligent Farm.

\section{Introduction}

In the very dynamic and competitive market of this century, successfully running a business means to be able to take advantage of the most advanced techniques and technologies available, achieving, by their appropriate integration with more classical approaches, a maximum of efficiency. Information Technologies (IT), control and communication tools may offer extremely important opportunities, but they usually are not very open for non-professionals, as business decision-makers are.

Cyber-Physical Systems, that represent the next generation paradigm for engineering systems, are more and more effectively being adopted in industry, being expected to offer the means for modelling, managing and connecting heterogeneous systems $[7,15]$.

In this context, there is a need for a generic framework allowing the design of enterprise architectures that can tightly couple business and production models, as well as the capability to extend towards complex business systems - which represents the main objective of this paper.

The paper is structured in 6 sections. Section two addresses the paradigm of Cyber-Physical Systems both from a multidisciplinary point of view, as well as a review of existing CPS architectures. Section three addresses the main problems in modelling Cyber-Physical Systems. In section four the authors propose a Conceptual Framework for Cyber-Physical Systems. The focus of the framework is related to systems modelling and information routing. The framework provides a unified view, integrating modelling and management from business level to production level with the aid of Cyber Physical Systems principles. Section five addresses a case study related to the implementation of the proposed framework in the field of agricultural enterprises [9].

\section{Cyber-Physical Systems - A Multidisciplinary Vision \\ 2.1 Cyber-Physical Systems Principles and Paradigms}

Cyber-Physical Systems represent an integration of computation and physical systems. The focus of such systems is to bring together the actual physical objects and the omnipresent computing and networking systems. CPS are concerned with the actual interaction between the physical and computer systems, thus completing the up to date research for understanding of the systems separately [5].

An important paradigm in close relation to CPS is the Internet of Things (IoT) which emphasizes the use of existing technologies and architectures for large-scale systems in order to identify and virtualize physical devices. Internet of Things components include: IoT Device Management, IoT Analytics, IoT Security, Event Stream Processing, IoT Platforms, IoT Standards and Ecosystems to create a real intelligent integrated system [6]. 
Another important emerging paradigm is Industrie 4.0, which $[1,22]$ specifically takes into account: sensor and actuator networks, intelligent network control systems and human in the loop principles; Intelligent Robots and Machines, including human-robot interaction, adaptive control, context awareness; Big Data; Network Quality of Service; Energy Efficiency And Decentralization; Virtual Industrialization in regard to the concept of "virtual plants and products"; Value Networks aiming at achieving digital integration along the supply chain and along different manufacturing processes and engineering models and methods $[7,18]$.

In this context, embedded systems that control physical processes can be viewed as a sub-set of the wider CPS definition.

An enterprise can be characterized as a complex system in a state of relatively stable equilibrium among many interdependent component systems in a shared human endeavour. In this context, the Intelligent Cyber Enterprise may represent the next generation of enterprises $[7,8]$.

Intelligent Cyber-Physical Systems (ICPS) represent the next generation of advanced networked systems, presenting distributed intelligence. Internet of Things and Internet of Services, integrated by multiple feedback loops, allow to develop complex cyber-physical systems [8].

As presented in Figure 1, CPS evolve from complex interactions of embedded systems (controls on board vehicles, smart intersections, communication networks, etc.), based on the networking and integration of them and involving human-technology interaction in application processes $[15,23]$.

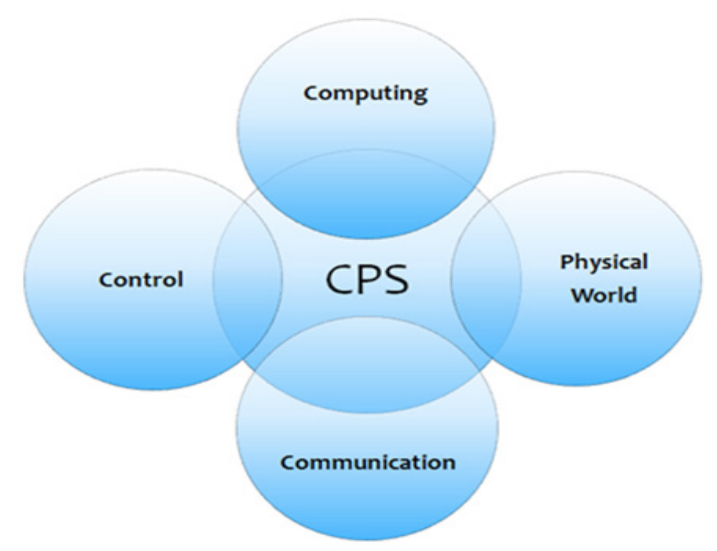

Figure 1. CPS as a multidisciplinary view
Communication between the system components is another important issue that needs to be taken into consideration. Cyber-Physical Systems need a real-time communication network that can provide guarantees on both the throughputs and delays of the system flow.

The communication network allows the formation of multiple control loops within its structure/ topology, so that multiple control objectives can be achieved at the same time. The location of the systems and the time difference between entities involved in the control loop are two essential issues caused by the distributed nature in CPS.

In this context, when modelling a Cyber-Physical System, one must take into consideration all the mechanisms that support semantic addressing for portability and reusability of applications, as well as system management. Also, a middleware framework is required to provide the right mechanisms which support the timelines behaviour on the control system and to tolerate faults. The middleware framework is useful to develop and control all the systems which involve the features of both heterogeneity and distributed operation.

From a networking viewpoint, some widelyrecognized characteristics of CPS can be outlined as:

- Network Complexity - the network of a typical CPS is often large in scale due to the number of existing distributed nodes;

- Resource Constraints - the embedded devices are limited in energy efficiency, bandwidth, rate through, throughput, etc.;

- Hybrid Traffic and Massive Data - due to the number of sensor and nodes, a large volume of data is created;

- Uncertainty - In CPS there are many factors that can cause uncertainty - sensor measurements error, computational model error, software errors, and unreliability of wireless communications or changes in network topology;

- Structural and behavioural complexities are more challenging for modeling, analysis, design, verification and implementation of CPS.

Future systems must be based on composition frameworks and more systematic or automated methods for proving connectivity. The complexity and heterogeneity of CPS impose special methods 
and tools to verify and prove the correctness of the overall system design. An overview of all aspects connected with this new paradigm is presented in Figure 2.

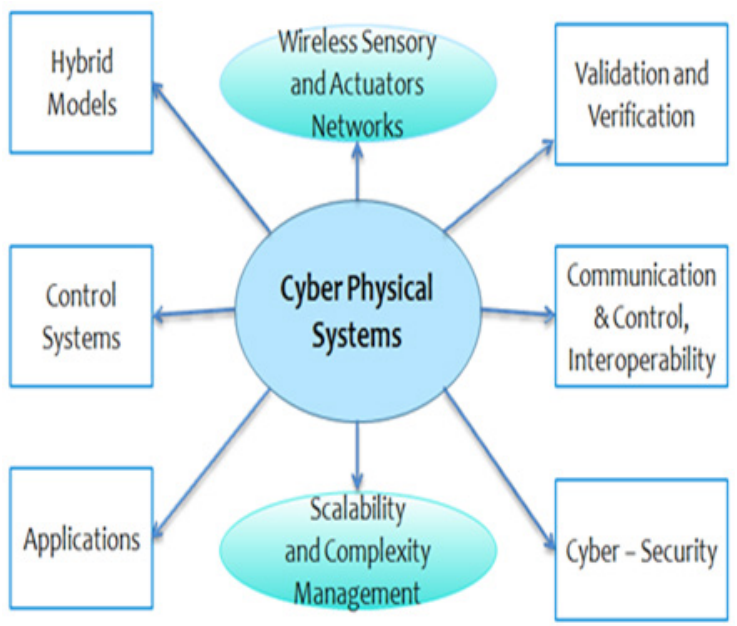

Figure 2. Main components of a Cyber-Physical System

\subsection{Models and Architectures in Cyber Physical Systems}

A series of CPS models, frameworks and generic architectures have been identified in literature.

The CPS 5C level architecture [16], proposes a 5 layer approach consisting of: Connection; Data Conversion; Cyber; Cognition and Configuration. The architecture allows for the development of complex systems starting from Monitoring Components towards Decision Support Systems and Resilient Control Systems. The 5C approach offers guidelines for the implementation of CPS in manufacturing industry.

The NIST - CPS Public Working Group proposes a CPS Framework oriented towards unifying the existing concepts as well as extending with specific topics. The framework addresses domains, concerns, properties, facets and aspects. A conceptualization facet, a realization facet and an assurance facet are proposed. The analysis process proposed encompasses the following steps: identify domains, identify cross-cutting concerns, analyse and address concerns [2].

A related concept refers to the liquid enterprise and the osmose concept developed in the OSMOSE project [10] The osmose concept envisions a semi-permeable membrane separating the real, digital and virtual environments. The OSMOSE architecture proposes a middleware system that enables intelligent communication supported by osmosis and context management. To enable the communication an inter-world router is proposed.

Reference Architectural Model Industrie 4.0 RAMI 4.0 [11] is an architectural model related to the Industrie 4.0 paradigm. In a structured manner RAMI 4.0 adopts a cube based layered representation including 7 Hierarchy Levels, a Life Cycle Value Stream addressed with the help of 6 Layers: Business, Functional, Information, Communication, Integration and Asset. The architecture aims at integrating Industrie 4.0 related concepts such as: Flexible production systems, networked systems, hierarchy based interaction, network integrated product [11].

The Reference Architecture for CPS-Platforms CYPROS - envisions an increase in productivity along with the implementation of intelligent systems. The reference architecture is composed of an information model a methodology for the description of architectural elements and a procedural model. The information model provides a framework for the developer to model CPS components.

\section{Main Problems in Modelling Cyber-Physical Systems}

The main challenges in order to develop the next generation of intelligent Cyber-Physical Systems are:

- High complexity of systems created by interaction of different heterogeneous objects with discrete and continuous behaviors;

- Need to integrate computing systems with physical processes and networks into complex systems;

- The interaction between physical processes, computing, hardware and software and communication networks requires the compositionality of the components and systems.

Having in mind the main characteristics and components of the CPSs, some technologies that facilitate abstract specification, conceptual modelling and virtual development, implementation and simulation could be considered: Abstractions and conceptual models; Logical frameworks and architecture; Functional and control models; Protocols, languages and standards. 
The dynamics of CPSs is complex, involving the stochastic nature of communication systems, the discrete event-driven dynamics of computing systems and the continuous one of control systems. Formal mathematical and information model of CPSs must be based on multi-level abstraction to consider overall structural and behavioural dynamics. The mathematical modelling of complex and heterogeneous systems must solve the semantic dichotomy between the continuous and the discrete event driven operation.

One possibility to model complex dynamics of CPSs is to use hybrid automata that can capture both the transition between discrete states and the evolution of continuous states over time. By using the Model of Computation (MoC) to define the rules of interaction between actors it is possible to create a simple hierarchical model. Considering different MoCs including Discrete Events (DE), Continuous Time (CT), Finite State Machine (FSM), Synchronous Reactive (SR), Process Networks (PN) and several varieties of dataflow could be developed the hierarchical composition that combine CT models with $\mathrm{DE}$ and FSM to model hybrid systems. The functional and structural models of different components must be completed with the implementation details such as the underlying platform and the communication networks [3].

The Model- Based Design (MBD) must take into consideration three main requirements:

- Compositionality: the property of a system to compute from local properties;

- Composability: the component properties are not changing as a result of interactions between components;

- Heterogeneity, in terms of the properties that are composed and their semantic domain represent the common future of all computational design frameworks. CPSs are inherently heterogeneous in structure, component behavior and types of interactions; they have three different design layers: physical, software and network/platform respectively.

The controllers and the desired dynamics of the physical processes are modelled first with physical layer abstractions, but the controller implemented is modelled using software layer abstractions. The software system is deployed on a computation platform modelled with network/platform layer abstraction.

The different abstraction layers use fundamentally different semantics and composition concepts into multi resolution hierarchical architectures.

The physical layer embodies physical objects and the interactions of them. On this level the mathematics of composition is based on linear algebra, topology and analysis and behaviour of physical object is determined by physical laws.

The software layer comprises the software components with behaviours expressed in logical time and whose interactions are modelled by various models of computations (MoCs).

The network/platform layer comprises the hardware side of CPS architectures (network architecture and computation platform that interact with the physical components by sensors and actuators).

Uncertainty in wireless communication must be mitigated by using the complex protocols to improve fault tolerance but the time varying delays introduced in transmission could destabilize the control loops [17, 21]. Trust, security and privacy (TSP) of Wireless Sensor Networks needs sensor node security, cryptography algorithms, key management approaches, secure routing strategies and data aggregation [14].

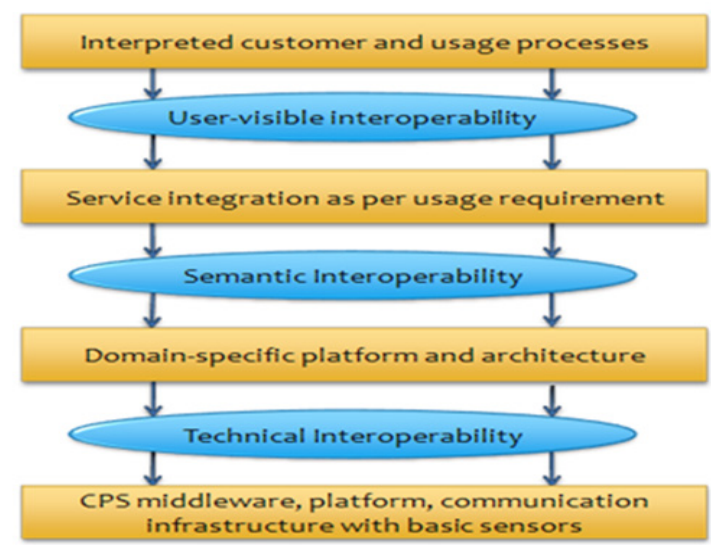

Figure 3. Main problems of CPS modelling

\section{A Conceptual Framework for Cyber-Physical Systems}

A starting point for the design of the conceptual framework is represented by the adoption of principles from proven enterprise architectures. 
In this context, authors propose a generic smart integrative platform, based on IoT concept and providing the user with a virtual, re-configurable ecosystem of services representing devices, embedded systems, networked control systems and smart objects, thus overcoming inherent fragmentation and heterogeneity, allowing an innovative approach in supporting multiple applications [25].

In order to fulfil its goals, the platform should consider the main aspects of heterogeneity, composability and compositionality at different levels of abstraction - systems, software architecture and controller level.

To allow modelling and analysing cross-domain interactions among physical and computational/ networking domains and to ensure predictability of the system behaviour, the platform will be designed at two main levels: abstract and functional.

The abstract level should integrate multi-domain models analysed in multiple levels topology and abstractions, multi-scale controls integrated in networked control systems [4].

At the functional level smart objects and platforms will be integrated in order to perform functionalities required by the business model conceived at the abstract level (Figure 4).

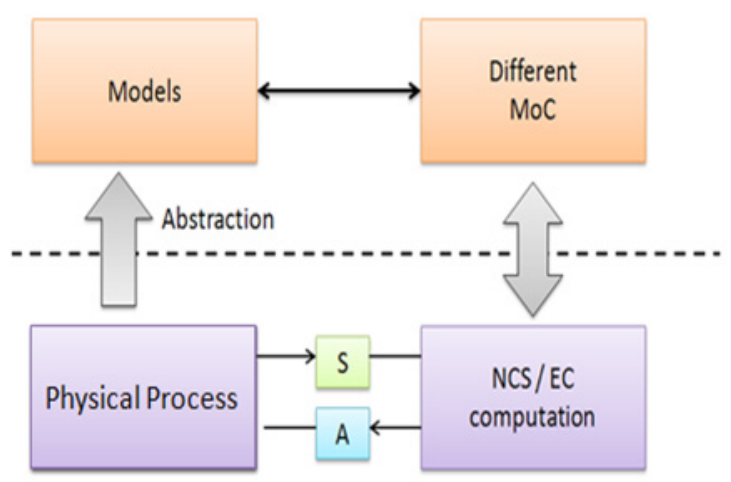

Figure 4. Integration of the main levels of the platform

The most difficult aspect will be the integration of these levels by mechanisms, languages and methodologies that will allow the platform to support developing applications, analysing and validating the performances.

As it will have to consider heterogeneity-different mathematical models, hardware and software, analogue and digital, cyber and physical, integrating different smart objects, the platform will have an Architectural Space and a Platform resources library defining components, composition rules, abstraction rules, refinement rules and interfaces that identify legal connections. From this point of view, interconnections and communication protocols will prove to be very important resources.

The complexity and diversity of heterogeneous components of the platform will be managed by generalized operators as Grouping, Focusing Attention and Combinatorial Search into intelligent manner. They will act especially at the abstract level and define a number of refinement steps that start from the initial description to the final implementation.

In this way it will be allowed the modelling of interactions, functionalities and implementation requirements, keeping model components consistent, preventing misconnection of Model Components. The platform will manage the system requirements and have the capacity to improve scalability and accuracy of model analysis including time and concurrency.

Other aspects that must be solved are related to the co-design of physical systems and controllers, orchestrating networked computational resources with physical systems considering time, concurrency in networked, distributed computational with physical systems. For such a goal, it will be necessary to define some general metrics to asses' verification and validation solutions.

The main characteristic of the proposed CPS architecture is represented by the need to define and model a CPS layer and to define and model the relation between the Business System and the Enterprise Environment in the context of CPS.

The proposed CPS architecture is composed based on a generic representation that can be extended by a set of associated views.

The first layer addresses the enterprise existing infrastructure.

The second layer addresses the enterprise as a CyberPhysical System.

The third layer addresses process management, Decision Support Systems and Data Analytics related to information associated to processes, products and by-products. 
The forth layer is addresses the business system, including: business intelligence and supply chain management.

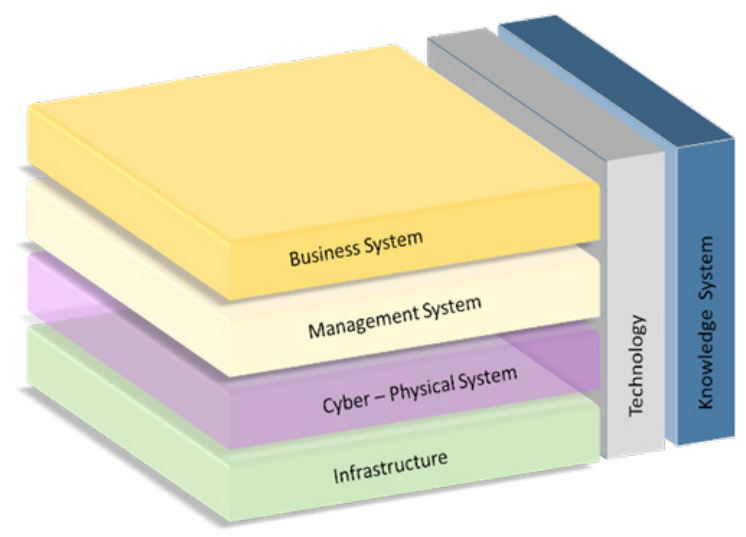

Figure 5. Generic Architecture

The infrastructure layer includes components such as equipment, machines, and resources.

The infrastructure system organized using infrastructure as a service principle facilitates the description and access to equipment, machines as well as sensing and actuating devices [20].

The Cyber-Physical System layer includes the following components: modeling, data acquisition, control, actuation, management and communication. This representation allows for the development of specific system architectures, data architectures as well as the connection with infrastructure architecture.

The main considerations related to the modelling component include:

- Models with solve - dependent, nondeterminate, or Zeno-behaviour (CPS can be seen as a hybrid systems using continuoustime models for physical processes and state machines, dataflow models, synchronous/ reactive models and/or discrete event models for computation);

- Keeping model components consistent (Multiple models with multiple variants of components must be integrated into complex systems) [18];

- Preventing misconnection of model components (Errors that appear within interconnected components must be taken into consideration, as well as unit errors, semantic errors, transposition errors) [11];

- Modelling of interactions of functionality and implementation (The dynamics of software and networks should be modelled accordingly); Modelling of
emergent behaviors.

distributed

and

The main considerations related to the data component include: data modelling, data interoperability, data storage [13].

The data architecture allows for the acquisition of sensor data, the integration of business related data and the semantic enhancement of data with the aid of metadata.

The main considerations related to the control component include:

- Networked Control Systems encompassing communication between controller and physical systems as well as between controllers;

- The integration of Hybrid Systems;

- $\quad$ Real Time Computing.

The main considerations related to the management component include: Management of distributed systems; Management of Hybrid Systems; Real Time Scheduling; Cyber Physical Systems Security.

The management component extends:

- Sensing capability: data related to the physical or virtual environment [24];

- Identity: ability to differentiate from other entities;

- Network: ability communicate the acquired data;

- Action capability: shaping the environment.

The CPS layer is directly connected to the Infrastructure layer and describes and manages all the entities that collect data from the physical environment (sensors), interact with the environment (actuators) and relay the data and commands to the upper level.

The following requirements need to be addressed in order to design the CPS layer: Agility, adaptability in production systems; autonomy and flexibility in production systems; Selfconfiguring capabilities adding or removing new resources or creating new task; Self-optimizing capabilities to improve its performances, by goal-directed control.

An open environment will be created for standardization and validation of models and complex systems interaction [18]. 
The general framework will allow identification and modeling of the different behaviours by connecting models and will generate a global autonomous behaviour. It will include specialized performance indicators and metrics for verification and validation of different functionalities, system security and reliability of different hardware and software architectures. In this direction, networks of sensors and actuators are integrated in order to verify new functionalities like: state awareness, real-time closed loop control, demand and incident management (Figure 6).

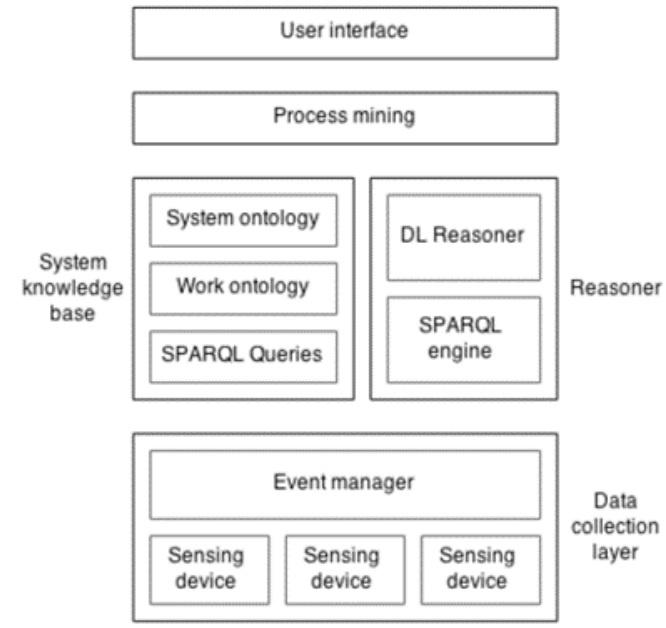

Figure 6. Generic system architecture [12]

Based on the CPS Architecture Framework, a Federated Web of Platforms Architecture focusing on CPS perspectives is divided in specific modules characterized by:

- Open architecture in order to facilitate the integration of future generation of technologies and embedded systems and collaborate with vendors and service providers; scalable storage capacity based on cloud computing solutions; management of system performance in order to ensure cost and energy efficiency;

- API platform with a rich set of builtin functions; standard-based message queue technology for existing IoT systems and platforms; definition of data models and acquisition of data form heterogeneous devices;

- H2M connectivity; cognitive integration; user friendly interface; M2M connectivity services to facilitate device integration and semantic interoperability; dynamic expendability in order to facilitate the integration of systems of systems; decision making support.
The Management System refers to process management, human resources, supply chain, decision support and business analytics.

The process management component includes: process analysis, process modelling, process optimization, process interoperability.

The Business System layer refers to the business vision, models and relations established between entities.

There is an explicit need addressed by the presented architecture to interconnect business models with process models as well as Cyber and Physical Models in order to optimize core activities in accordance with business needs. The envisioned system allows for a dual view in both virtual and real environments enhancing simulation and merging business analytics and process optimization.

The architecture incorporates the following views:

- Organizational view including vision and values;

- Process Management view including processes, entities, flows, transformation and exploitation of data;

- Socio - economic view including social organization systems and micro and macro socio-economic models;

- Technological view including the technologies.

\section{Case Study: The Agricultural Enterprise as a Complex Cyber - Physical System}

Current Industrial Revolution driven by CPS and IoT is expected to have a major impact on the future of agriculture as well, as there is a natural relation between industry and agriculture [1]. As a extension of Industry 4.0 a new concept can be introduced: Agriculture 4.0. Integration of machines and equipment, increased automation, efficient decisional process represents objectives of an agricultural enterprise facilitating the adaptability to climate and market dynamics and perturbations and allowing for sustainable, ecological and socially beneficial development.

When addressing the agricultural enterprise concept (which will be referred subsequently, in short, as "farm") organization we must take 
into consideration the following agriculture related concepts [7]: Sustainable and conservable Agriculture, Organic Agriculture, Industrial Agriculture, as well as Community Supported Agriculture.

Also, in accordance with an EU Agricultural and Rural Development Study "Precision Agriculture (PA) is a whole-farm management approach using information technology, satellite positioning (GNSS) data, remote sensing and proximal data gathering. These technologies have the goal of optimizing returns on inputs whilst potentially reducing environmental impacts." [4].

In Figure 7, a vision of farm components is presented, focusing on resources, processes and products. The general farm representation is assumed considering both animal and vegetal products and, consequently, the three major components that need to be need to be addressed when considering a paradigm shift in farm organization:

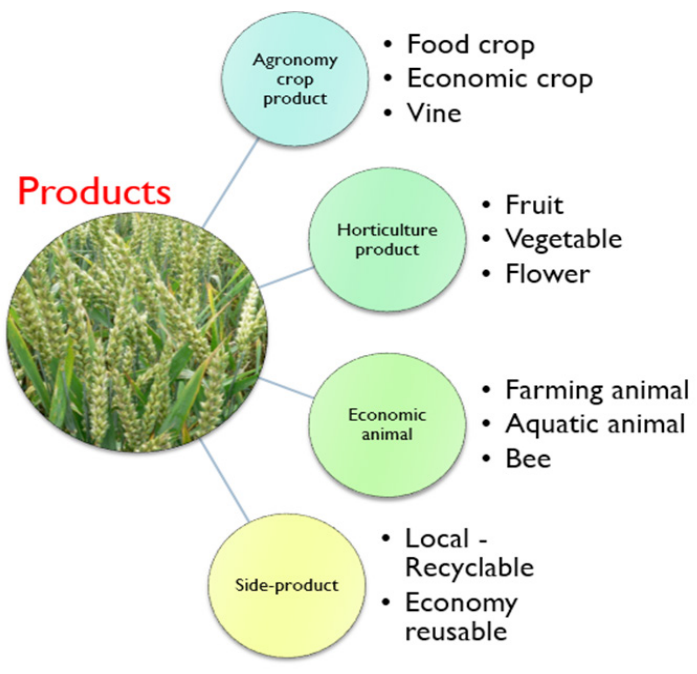

Figure 7. Main components and activities of a generic farm

Integrating and processing the data regarding the crops, the soil and the agricultural production can and should assure the efficient exploitation of the agricultural fields, the soil and an optimal hydration through a real-time intelligent control system.

Agriculture organizations need to address the problem of complex systems in relation to emerging paradigms such as Internet of Things and Cyber Physical Systems. CPS are very important in assuring optimal control, by connecting intelligent sensor networks.
Also, in the logistics field, new opportunities arise in the area of intelligent objects, ensuring the optimal level of real-time monitoring of the objects' state and place.

Integration CPS in Agriculture Organizations must take into consideration:

- Components heterogeneity and complexity that require new models and representation languages in order to allow interoperability and composition;

- Natural agents interaction (human operators) with hardware and software agents;

- Uncertainties existing in complex systems, generated by the physical processes and communication networks;

- Performance measuring and validation systems, taking into consideration new metrics.

We can identify three major farm subsystems in accordance with the product type:

- The soil management subsystem, which includes all the general aspects related to culture and pasture planning, fertilization and soil humidity monitoring and control, with respect to actual conditions, weather forecasts, market predictions, enterprise goals and production techniques and technologies;

- The plant management sub-system, which deals with the implementation of goals settled by the soil management sub-system for crops; it takes into account aspects related to plant selection, work planning in accordance with the crop specificities and soil quality and updated according with weather conditions, plant health and so on;

- The livestock management sub-system, with similar goals, but related to animals.

Smart Farming includes the following areas of interest: Sensor system, Traceability, Smart agrilogistics, Smart food awareness.

By using a suitable selection of machines, an increased automation degree, efficient exploitation of multi-annual databases in the decisional process, the objective of an agricultural enterprise, with real capabilities of self-reconfiguration and adaptation at environmental, weather and market dynamics and perturbations, can be achieved.

The architecture of the agricultural enterprise is composed of a generic representation and a set of views associated to the representation. 
It is represented in Figure 8, and is comprised of building blocks encompassing relevant concepts for the farm organization. The building blocks are organized in tiers and detailed with the aid of architectural views.

The first tire encompasses the farm existing continuum and is represented by the following aspects: pre-product, infrastructure, resources and farm human resources.

The second tier is represented by acquired data and existing or necessary know-how.

The third tier is represented by processes, information associated to processes, products and by-products, and farm systems.

The forth tier is represented by the management system, business intelligence and decision making, knowledge management and supply chain management.

The Farming machines subsystem addresses:

- Traffic management: driver assistance, vehicle movement tracking and operations tracking, process tracking, seeding and treatment tracking [19] [12];

- Operational planning.

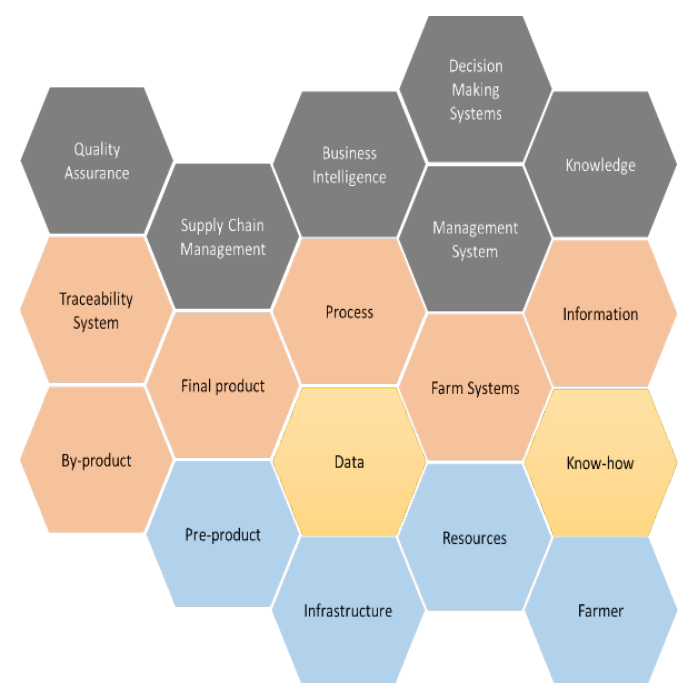

Figure 8. Generic Farm Architecture

The Farm data acquisition system includes: crop monitoring, soil monitoring, livestock tracking and monitoring, weather monitoring, biomass monitoring, farm parameters sensor monitoring, machine vision systems, remote sensing (drones and robots).
The Farm Automation System include: irrigation systems, feeding systems, other specific automation systems.

The IoT layer is directly connected to the Physical Sub-layer in order to collect data from sensors, interacting with the environment and relay the data to the upper level (communication): Sensors, Actuators, Wireless sensors networks (Figure 8).

\section{Conclusions}

A generic architecture based on Cyber-Physical Systems concept has been proposed using a predefined scenario.

A smart farm can be implemented by integrating the most important results of the research in advanced technological fields. Thus, CyberPhysical Systems play an important role, seen from a multidisciplinary perspective.

\section{REFERENCES}

1. Blanchet, M., Rinn, T., Thaden, G., Thieulloy, G. (2014). Industry 4.0: The new industrial revolution-How Europe will succeed, $\mathrm{Hg}$. v. Roland Berger Strategy Consultants GmbH, München.

2. Cyber Physical Systems Public Working Framework for Cyber-Physical Systems, Release 1.0, May 2016.

3. Derler, P., Lee, E. A., \& Vincentelli, A. S. (2012). Modeling cyber-physical systems. Proceedings of the IEEE, 100(1), pp. 13-28.

4. Dumitrache, I. (2010). The next generation of Cyber-Physical Systems, in Journal of Control Engineering and Applied Informatics, 12(2), ISSN: 1454-8658, pp. 3-4.

5. Dumitrache, I. (2012). Some Modelling and Design Challenges in Cyber Physical Systems, invited paper on ICSTCC 2012, IEEE Conference.

6. Dumitrache, I., Caramihai, S. I., \& Stanescu, A.M. (2013). From Mass Production to Intelligent Cyber-Enterprise, in 19th International Conference CSCS, IEEE, pp. 399-404. 
7. Dumitrache, I. (2014). Cyber-physical systemsnew challenges for science and technology, in Journal of Control Engineering and Applied Informatics 13.3, ISSN: 1454-8658.

8. Dumitrache, I. (2014). Intelligent CyberEnergy-Systems - invited paper on ICTSCC18th International Conference on System Theory, Control and Computing.

9. Dumitrache, I. \& Caramihai, S.I. (2015). Agricultural Enterprise as a Complex System: A Cyber Physical Systems Approach, in 20th International Conference on Control Systems and Computer Science (CSCS), pp. 659-664.

10. Gusmeroli S, G. Monteleone, C. Agostinho C. Lucena, M. Sesana, A. Felic, K. Fischer. Osmose: A paradigm for the liquid-sensing enterprise. Proceeedings of the Workshops of the IWEI 2015.

11. Hankel, Martin, and Bosch Rexroth. „The Reference Architectural Model Industrie 4.0 (RAMI 4.0).” ZVEI (2015).

12. Jardim Goncalves, R., Sarraipa, J., Agostinho, C.A. \& Panetto, H. (2011). Knowledge framework for intelligent manufacturing systems, in Journal of Intelligent Manufacturing, Volume 22, Issue 5, Springer US, pp. 725-735.

13. Jardim Goncalves, R., Grilo, A. \& Popplewell, K. (2014). Novel strategies for global manufacturing systems interoperability, in Journal of Intelligent Manufacturing, 1-9, Volume: 27 Issue: 1, Special Issue: SI, pp. 1-9.

14. Kewei, S. \& Sherali, Z. (2015). Data Quality Challenges in Cyber-Physical Systems, in Journal of Data and Information Quality, Volume 6 Issue 2-3, July 2015, Article No. 8, ACM New York.

15. Kim, K. D., \& Kumar, P. R. (2012). Cyber-physical systems: A perspective at the centennial. Proceedings of the IEEE, 100(Special Centennial Issue), 1287-1308.

16. Lee, J., B. Bagheri, and Hung-An Kao. „A cyber-physical systems architecture for industry 4.0-based manufacturing systems." Manufacturing Letters 3 (2015): pp. 18-23.

17. Park, J., Inwhee J. \& Won-Tae K. (2014). An Efficient Discovery Protocol of Large-Scale CPS Middleware for Real-Time Control System, in Studies in Informatics and Control, ISSN 1220-1766, vol. 23 (1).
18. Repta, D., Moisescu, M.A., Sacala, I. S., Dumitrache, I. \& Stanescu, A. M. (2015). Towards the development of semantically enabled flexible process monitoring systems, in International Journal of Computer Integrated Manufacturing, pp. 1-13.

19. Sacala, I. S., Moisescu, M.A. \& Repta, D. (2013). Towards the Development of the Future Internet Based Enterprise in the Context of Cyber-Physical Systems, in 19th International Conference on Control Systems and Computer Science, pp. 405-412.

20. Sacala, I.S. \& Moisescu, M.A. (2016). Towards the Development of Interoperable Sensing Systems for the Future Enterprise, in Journal of Intelligent Manufacturing, Print ISSN 09565515, Springer.

21. Sandberg, H., Amin, S. \& Johansson, K. (2015). "Cyber physical Security in Networked Control Systems: An Introduction to the Issue, in IEEE Control Systems, ISSN: 1066-033X, pp. 20-23.

22. Schuh, G., Reuter, C., Hauptvogel, A \& Dolle, C. (2015). Hypotheses for a Theory of Production in the Context of Industrie 4.0., in Advances in Production Technology, Springer, pp. 11-23.

23. Syed, U., Khalid, D, B., Brad, R. \& Bala Zamfirescu, C. (2014). Fast Edge Detection Algorithm for Embedded Systems, in Studies in Informatics and Control journal, ISSN 1220 1766, vol. 23 (2), pp. 163-170.

24. Vargas, A., Cuenca, L., Boza, A, Sacala, I.S. \& Moisescu, M.A. (2014). Towards the development of the framework for inter sensing enterprise architecture, in Journal of Intelligent Manufacturing, pp. 1-18, Publisher Springer US, ISSN 1572-8145.

25. Thorsteinsson Gisli, Gunnarsdottir Rosa, Niculescu Andrei, Assessing the Value of a Mobile Application in Fostering Ideation within a School Context, Studies in Informatics and Control, ISSN 1220-1766, vol. 24 (1), 2015, pp. 119-126. 